\title{
Robotic rock handling during backhoe excavation
}

\author{
X. D. Huang ${ }^{a}$ and L. E. Bernold ${ }^{b}$ \\ apepartment of Mechanical and Aerospace Engineering, Box 7910, North Carolina State \\ University, Raleigh, NC 27695-7910, USA
}

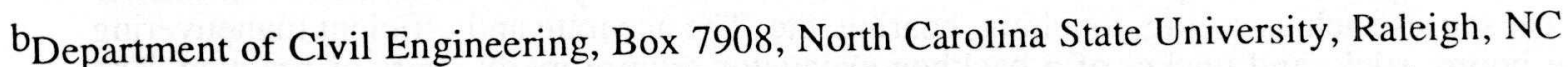
27695-7908, USA

\begin{abstract}
Excavation of soil and rock is a high volume and repetitive construction operation. Research in robotic excavation has been focusing mainly on path planning and path control. Very little is known about how to detect and handle underground obstacles such as rocks or utility lines robotically. This paper introduces a research facility to study robotic backhoe excavation using a heavy-duty hydraulically powered and computer controlled manipulator. Conventional excavation methods and an existing telerobotic excavator are introduced. The control system for this robotic backhoe excavator is based on multiple sensors for force, acceleration and position measurements. The same set of sensors are also used for obstacle detection, recognition and mapping. An approach for the detection and recognition of removable obstacle is discussed. Contour mapping procedures have been developed to provide a partial surface map of a buried obstacle.
\end{abstract}

\section{BACKGROUND}

\subsection{Introduction}

Almost all production oriented robots today are utilized within the manufacturing industry. One main reason why true robots are hardly seen in the construction environment is because of the existed unstructured and complex conditions. In addition, construction mostly takes place in an uncontrolled environment, exposed to the weather, dust and noise. The attributes of the materials to be handled range from large, heavy, bulky, and non-homogeneous to light, fragile, and homogeneous. In addition, although one of the traditional materials handled in construction is soil, the mechanics of excavating soil and rocks is poorly understood.

Despite the many difficulties, opportunities for applying high technology in construction are abundant. For certain applications and situations, such as construction in hazardous areas (i.e., nuclear waste disposal, space construction), robotic technology is unavoidable [1]. However, applying high technology in these areas requires empirical as well as theoretical research.

Excavation of soil and rock is a high volume and repetitive construction operation. It is estimated that a fully automated excavating machine performing at $25 \%$ the efficiency of an expert human operator would be commercially feasible [2]. The exploration of space offers still another challenge to excavation. NASA studies conclude that excavation will be one of the first activities necessary to establish planetary habitats [2]. Robots are expected to do much of the work. 
Studies on the applications of robotic excavation have been undertaken by several researchers. Vaha in 1990 studied and established a kinematic and dynamic control model for a robotic excavator [3]. Bullock and Oppenheim in 1992 developed an approach for forcecognitive robotic excavation [4]. Tochizawa et al. in 1991 reported about an automated excavator for excavating a trench for drainage using laser guidance [5]. The authors showed that the laser guidance helped to decrease the labor hours and increase the digging accuracy.

\subsection{Conventional Excavation Method.}

Some $30 \%$ of the earth's crust is shale or mudstone, much of which can be excavated by using a backhoe, front-end loader, or other heavy mechanical excavators. Because of their versatility, backhoe excavators are especially popular on construction sites. During excavation, the equipment operator adjusts the digging angle, depth of penetration and length of digging in order to fill the bucket most efficiently under the given constraints. His/her hands and feet, necessary to operate the equipment, are coordinated intuitively and integrated with his/her physical senses such as seeing, touching, hearing, etc. The accurate and efficient maneuvering of the boom, stick, and bucket of a backhoe excavator require many years of practice. The handling of obstacles is yet another problem. Especially when interfering with an unexpected object, the operator's experience and craftsmanship are crucial in avoiding damages. Water mains, gas lines, or telephone cables are just a few of the obstacles which have to be avoided. In the case of a removable obstacle such as a rock, the operator has several options for dealing with it. He/she can a) remove the rock by loading it into the bucket, b) dig around the rock if it is too big, and c) push, slide/roll the rock from its original place. The operator analyzes the situation, decides what to do based on the gathered information (e.g., visual inspection) and executes the decisions on the basis of his/her previously established rules.

\subsection{A Teleoperated Excavator}

Langreth reported about an advanced teleoperated hydraulic excavator - Haz-Trak by Kraft TeleRobotics [6]. This excavator operates with a master-slave control and incorporates the force-feedback control so that the human operator can not only control the mechanical arm very easily, but also "feel" the obstacles that the bucket hits. Provided with video images from three cameras, the operator sits remotely inside a building or a vehicle, manipulating and observing the operations. The Haz-Trak incorporates many features useful for cleaning up hazardous chemical and radioactive wastes at weapons labs or removing unexploded munitions buried at military installations. Because of the force sensors inside the hydraulic valve manifold, resistance at each joint are measured and transmitted back into the controller. This enables the operator to probe for buried obstacle and waste without accidentally puncturing a barrel and releasing radiation or toxic chemicals.

\section{AN EXPERIMENTAL FACILITY FOR ROBOTIC EXCAVATION}

A Multipurpose Robotic Manipulator Platform (MRMP) has been built within the Construction Automation and Robotics Laboratory of North Carolina State University. This robotic manipulator has a reach of 6 meters. It is driven by one hydraulic motor for base rotation and three hydraulic actuators (cylinders) which provide a total of four-degree-offreedom (DOF). Figure 1 depicts an overview of the experimental device. Many different end effectors (i.e., bucket, gripper) can be mounted at the end of the arm to reassemble this device into different experimental configurations. Presently, the MRMP is equipped with a bucket to operate as a computer controlled backhoe excavator.

Two kinds of sensors are utilized in the data collection system. One force sensor - load cell, is mounted at the rod of the third hydraulic actuator. Three accelerometers are mounted on the boom, the arm and the end-plate (connection between the bucket and arm) respectively to 
detect accelerations both in the digging plane and perpendicular to the digging plane (it is assumed that the base does not rotate during one digging path so that movements of the boom, arm and bucket stay in one plane). A metal detector search coil will be adopted to mount on the bottom of the arm to detect some utility lines and electric wires. By using an analog/digital board with a sampling rate over $4 \mathrm{~K} \mathrm{~Hz}$, the 386 computer reads at real-time the changes from the force sensor and the accelerometers. Control commands from the $386 \mathrm{PC}$ computer are sent to the actuators through a digital/analog board. The commands act on four electrohydraulic proportional flow control valves.

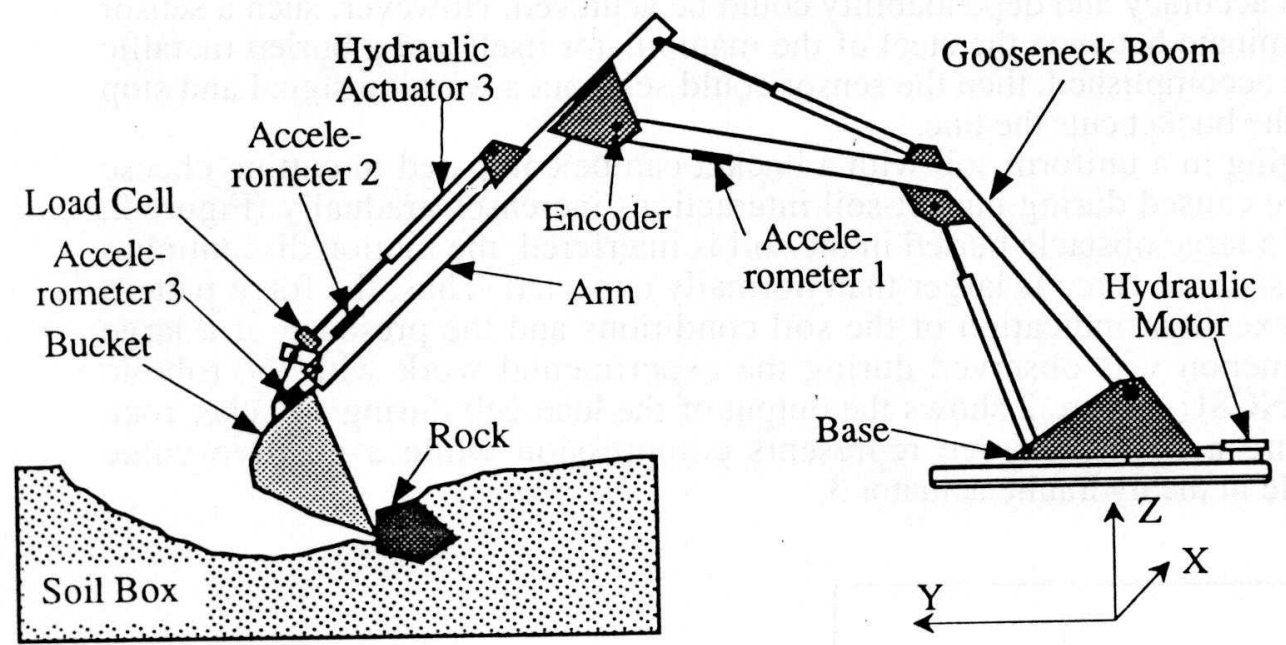

Figure 1. Experimental facility for robotic backhoe excavation

\section{IN PROCESS OBSTACLE DETECTION AND RECOGNITION}

\subsection{Background}

Obstacles encountered during excavation can be divided into two basic categories: 1) removable rigid obstacles like rocks or lumber pieces, and 2) non-removable objects like utility lines. In 1989, the United Kingdom reported about 70,000 instances of damage to buried services during excavation [7]. It becomes more and more critical that during excavation, underground buried obstacles such as utility lines can be detected and distinguished from removable obstacles such as rocks to avoid accidents.

However, tasks which humans find simple and trivial are sometimes complex and difficult for a robot to perform. A human can find an arbitrary object visually in a cluttered environment and proceed to grasp the object and move it at will, avoiding obstacles along the way. This task is beyond the capability of most robots in use today.

One approach to building robots for tasks such as object recognition, manipulation, and collision avoidance is to study human performance, hoping to gain insight into the interaction of human sensors, cognition, and motion control. This is still an active research area, but results directly applicable to actual present day robotic tasks are few [8]. A second approach is to exploit existing technology. Most of the researchers are focusing on the applications of 3dimensional vision systems and tactile sensing devices, which depend on expensive and sensitive equipment. However, construction usually takes place in a harsh environment where 
rugged and dependable tools and devices are required.

Fortunately, a variety of other sensory devices have been developed and tested for heavyduty application. The following discussions present an idea of integrating several introduced standard industrial sensors for removable obstacle detection and recognition.

\subsection{Detection of the Removable Obstacle}

Based on the above discussion, a robotic backhoe excavator has to be able to discriminate between utility lines and removable obstacles. One possible solution to this problem is the use of electromagnetic detectors capable of detecting metal pipes and electrical wires. By attaching such a sensor to the arm close to the actual location where the bucket will interfere with a metallic obstacle, high accuracy and dependability could be achieved. However, such a sensor must be able to discriminate between the steel of the manipulator itself and a buried metallic object. If this could be accomplished, then the sensor could send out a warning signal and stop the excavation before the bucket cuts the line.

The action of digging in a uniform soil with a bucket can be compared to cutting cheese with a knife. The force caused during bucket-soil interactions increases gradually (Figure 2, before 75 samples). If a large obstacle buried in the soil is interfered, the motion discontinues when the measured resistance force is larger than normally expected. Thus, the force pattern during digging is an excellent indication of the soil conditions and the presence of a large obstacle. This phenomenon was observed during the experimental work with the robotic backhoe excavator at NCSU. Figure 2 shows the output of the load cell during a bucket-rock collision. In this figure a negative force represents compression while a positive value represents tension mode in the hydraulic actuator 3.

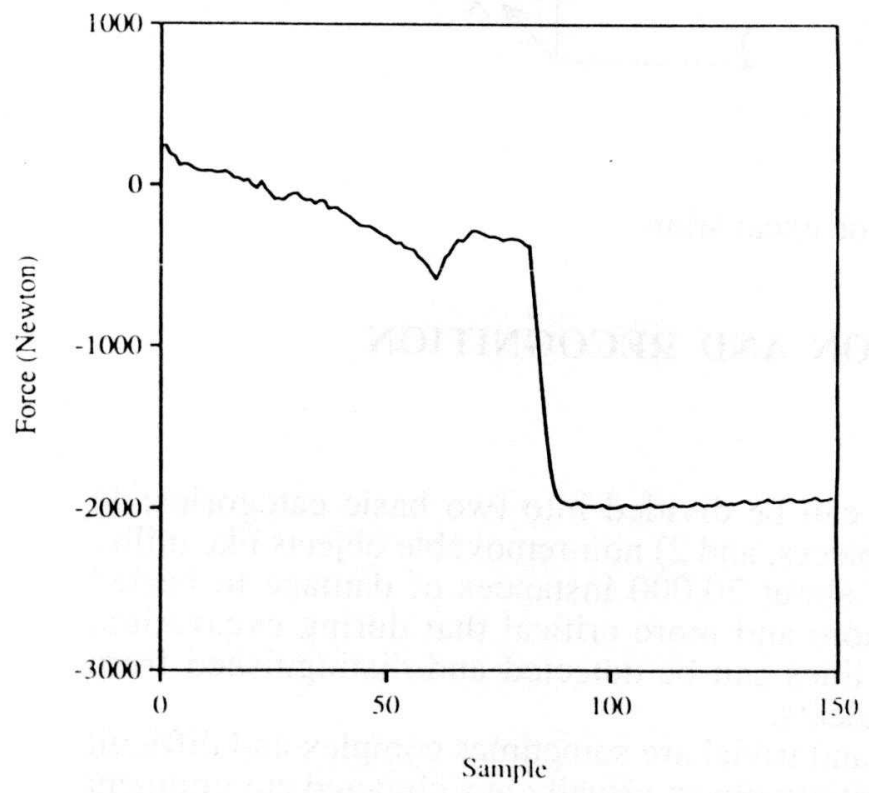

Figure 2. Load cell force pattern during bucket-rock collision

Figure 2 shows that after the compression force undergoes a normal increase, an abrupt and drastic increase can be observed. Actually at exactly this point, the bucket hit a buried rock in the soil box. The slope of the curve at this point is almost vertical. As the compression force reaches $-2000.0 \mathrm{~N}(-450 \mathrm{lbs})$, the control system would then stop the excavation motion and switch to the obstacle handling mode. The impact position will also be recorded in the computer data base. The horizontal line in the force profile then indicates a stable force. 


\section{PARTIAL SURFACE CONTOUR MAPPING}

\subsection{Surface Representation}

While it is important to know the presence of an obstacle in the digging path, more information is needed before a decision can be made about how to handle the buried object. If the obstacle is known not to be part of a utility line, it is still not clear if it is removable at all. In other words, an encountered rock surface could actually represent bedrock. Thus, it is necessary to acquire more data to describe the obstacle, particularly its dimension. As mentioned earlier, vision sensors would have great difficulties in providing this information since the obstacle is mostly buried and covered with the soil. The tactile sensing concept has been chosen as a first approach to develop a contour surface map of the obstacle.

The obstacle's surface can be represented by $\mathrm{X}, \mathrm{Y}, \mathrm{Z}$ coordinates of all contact points. Figure 3 shows a parametric representation of a surface patch $\mathbf{P}(u, v)$, where the surface parameters $u, v$ range from 0 to 1 . The patch has four knot points, $\mathbf{P}(0,0), \mathbf{P}(0,1), \mathbf{P}(1,0)$, $\mathbf{P}(1,1)$ which determine the boundaries of the patch.

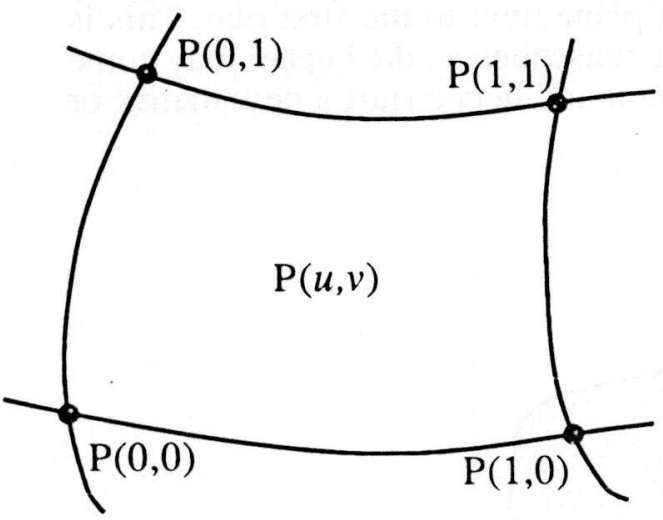

Figure 3. Parametric surface patch

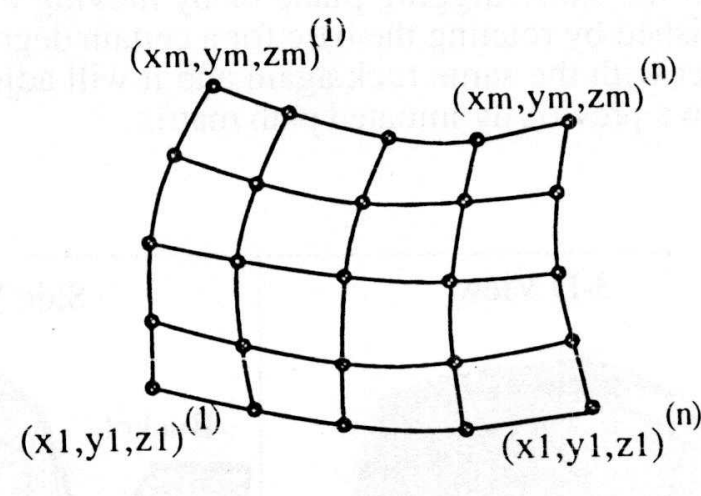

Figure 4. Composite surface with $\mathrm{x}-\mathrm{y}-\mathrm{z}$ coordinates

The knot points are obtained from the position sensors (e.g., joint encoder) while the bucket makes tactile contact with the obstacle. The notion of a single patch can be extended, as more and more data will be available, to a composite surface containing a series of curvaturecontinuous patches defined on an arbitrary knot set (Figure 4). These data or knot points first need to be organized before further surface mapping can be built up. In the obstacle surface contour mapping experiments, the collected data are put in a matrix format, which later can be used to generate key dimensions of the obstacle. For example, the following is a set of matrices which can be used to represent the surface patch(es) shown in Figure 4.

$$
\left[\begin{array}{c}
\mathrm{x} 1, \mathrm{y} 1, \mathrm{z} 1 \\
\mathrm{x} 2, \mathrm{y} 2, \mathrm{z2} \\
\vdots \\
\mathrm{xm}, \mathrm{ym}, \mathrm{zm}
\end{array}\right]^{(1)},\left[\begin{array}{c}
\mathrm{x} 1, \mathrm{y} 1, \mathrm{z} 1 \\
\mathrm{x} 2, \mathrm{y} 2, \mathrm{z} 2 \\
\vdots \\
\mathrm{xm}, \mathrm{ym}, \mathrm{zm}
\end{array}\right]^{(2)}, \ldots \ldots,\left[\begin{array}{c}
\mathrm{x} 1, \mathrm{y} 1, \mathrm{z} 1 \\
\mathrm{x} 2, \mathrm{y} 2, \mathrm{z} 2 \\
\vdots \\
\mathrm{xm}, \mathrm{ym}, \mathrm{zm}
\end{array}\right]^{(\mathrm{n})}
$$

In the matrix representation, an $\mathrm{X}-\mathrm{Y}-\mathrm{Z}$ coordinate is used to define an impact point on the contact surface. One matrix records all the contact points within one digging plane (path). 
Therefore, several different matrices have more data points and may produce a partial surface description. The total set of $n$-matrices establishes a grid pattern of points (and patches.) Once the entire surface has been built up, the dimensions of the obstacle, such as its width and height can be easily derived.

\subsection{Partial Surface Map Derivation --- Analysis and Results}

Position and acceleration sensors are presently utilized in the derivation of the surface map of a rock buried in the soil box. A partial obstacle contour mapping algorithm has been developed and tested. It utilizes the force and position control principles to command the bucket tip around the obstacle while probing and storing the coordinates of the impact points. After a drastic and unexpected increase in force output, a mapping procedure is invoked that comprises five steps: 1) Identification which point of the bucket made contact with the obstacle, 2) storage of the impact position into the matrix of the path, 3) execution of a small back track motion, 4) movement of the bucket tip up vertically by $0.02 \mathrm{~m}$, and 5) continuation of forward motion parallel to the original trajectory. If the rock is hit again after the first adjustments, the above five-step mapping procedure is repeated until the obstacle will be bypassed at its top and the current digging path will be continued at a new level.

During the execution of one digging path, only one set of points is stored into a matrix. It is apparent that further data are needed in order to create the complete grid map either by digging deeper in the same digging plane or by moving to a new plane next to the first one. This is accomplished by rotating the base for a certain degree. As a consequence, the bucket may come in contact with the same rock again and it will adjust its path and hence start a new matrix or append to a previously initiated path matrix.

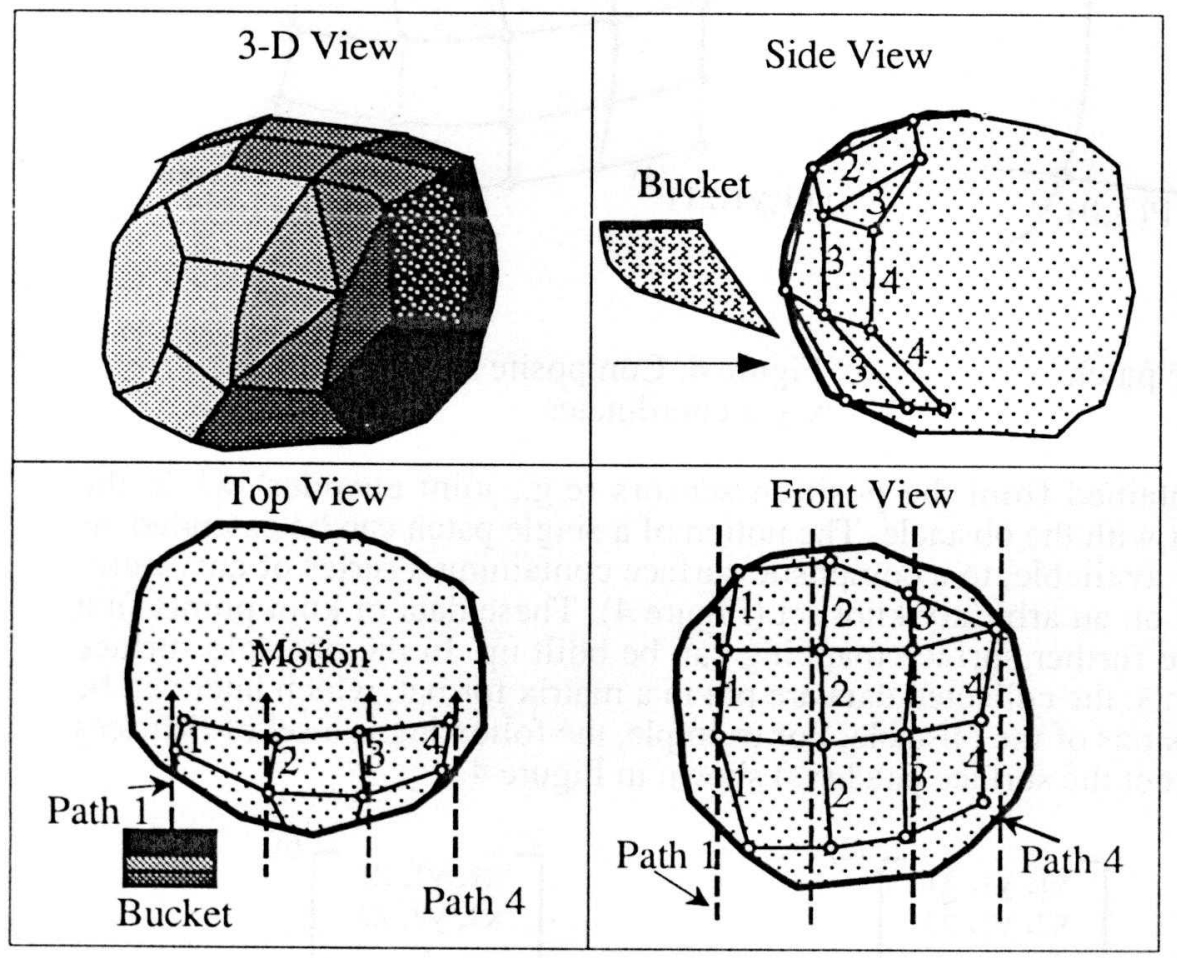

Figure 5. View-based surface grid maps of a hypothetical obstacle 
Figure 5 depicts a buried rock from different view points. The circles show the contact points while the grid lines indicate the interpolation web structure between the contact points. A total of four digging paths are necessary to map the surface. Each path is represented by a dashed line (see front view.) Since the bucket has a certain width, it may hit the rock at different places on its tip. One accelerometer has been installed to detect different deflections of the bucket caused by an eccentric force. By gathering data from that accelerometer, the actual point of impact on the bucket can be derived. Therefore, the actual impact position may vary from the path, as shown in Figure 5.

Figure 6 presents graphically the actual obstacle surface map derived through the above processes. Each line in the Y-Z plane represents a partial cross section and the actual trajectory followed by the tip of the bucket. Plotted on the $\mathrm{X}$-axis are the rotational advancements between the four paths. The Z-axis represents the vertical path adjustments. This partial surface map is built from the elements in the data matrices which contain those coordinates where the bucket collided with the obstacle. The resulting matrices can be arranged as follows:

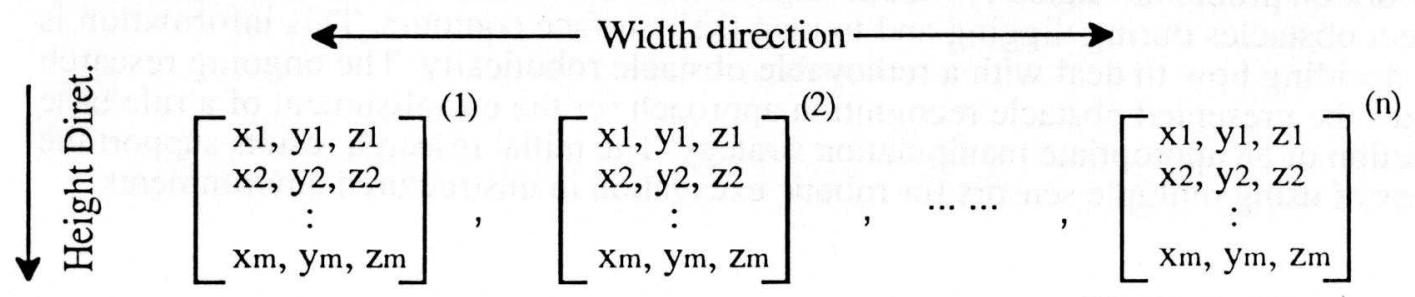

Each of these matrices represents a vertical plane (or path). It is very seldom the case that all the points in a matrix will be generated during one path alone, due to the fact that excavation progresses from top to bottom. Thus, one matrix may have to be appended with impact points which lie deeper then the original set. Sorting algorithm can be used to rearrange the matrices after the completion of the mapping procedure, which will enhance the recognition efficiency of border points in one plane.

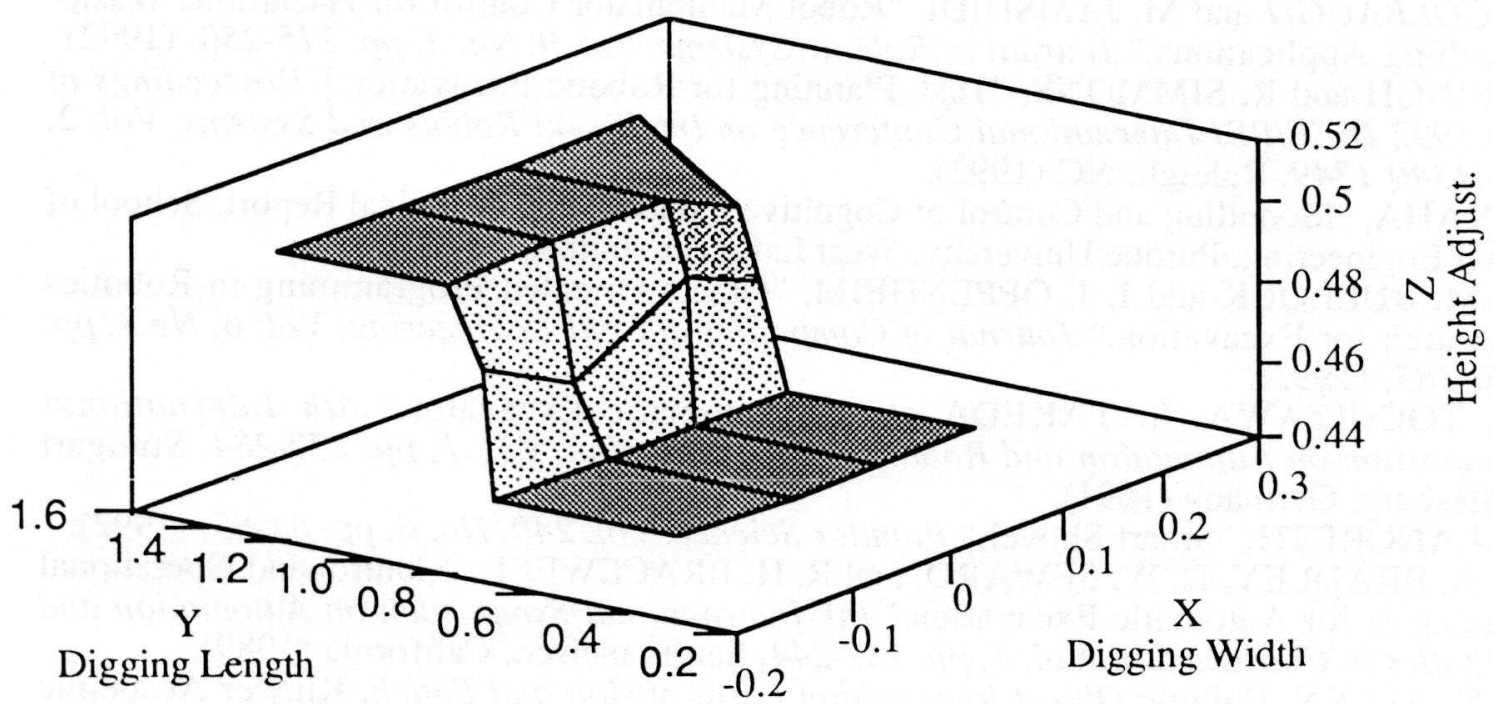

Figure 6. Partial surface map of a buried rock

A removable obstacle can be handled in a variety of ways, depending on the objective of the excavation and the characteristics of the obstacle itself (such as size, shape, etc.). Strategies 
have to be developed for this purpose. Finding the "best" strategy is a decision making process which may take advantage of a rule base using some input conditions. These conditions include: 1) the data about the dimensions of the obstacle, 2) the excavation requirements, 3) the mechanical system configurations, and 4) relative position of the bucket/excavator to the obstacle. Ongoing research in robotic excavation investigates different obstacle handling strategies that are using the information from the implemented contour mapping procedure.

\section{CONCLUSION}

This paper presents concepts and experimental results of research on robotic excavation, obstacle detection, and recognition. Multiple sensors such as a load cell, accelerometers, and angle encoders have been installed on a large-size computer controlled backhoe excavator for empirical work on problems related to robotic excavation. The control system is designed to be able to detect obstacles during digging and to map their surface contours. This information is critical for deciding how to deal with a removable obstacle robotically. The ongoing research effort utilizes the presented obstacle recognition approach for the establishment of a rule base for the selection of an appropriate manipulation strategy. The initia! research results support the effectiveness of using multiple sensors for robotic excavation in unstructured environments.

\section{ACKNOWLEDGMENT}

This research has been supported by the College of Engineering at North Carolina State University and the Space Studies Institute at Princeton, New Jersey.

\section{REFERENCES}

1 R. COLBAUGH and M. JAMSHIDI, "Robot Manipulator Control for Hazardous WasteHandling Applications," Journal of Robotic Systems, Vol. 9, No. 2, pp. 215-250, (1992).

2 S. SINGH and R. SIMMONS, "Task Planning for Robotic Excavation," Proceedings of the 1992 IEEE/RSJ International Conference on Intelligent Robots and Systems, Vol. 2, pp. 1340-1349, Raleigh, NC (1992).

3 P. VAHA, "Modelling and Control of Cognitive Excavation," Technical Report, School of Civil Engineering, Purdue University, West Lafayette, Indiana (1990).

4 D. M. BULLOCK and I. J. OPPENHEIM, "Object-Oriented Programming in Robotics Research for Excavation," Journal of Computing in Civil Engineering, Vol. 6, No.3, pp. 370-385, (1992).

5 M. TOCHIZAWA, S. TAKEDA et al, "Automatic Excavator," 8th International Symposium on Automation and Robotics in Construction, Vol. 1, pp. 277-284, Stuttgart Killesberg, Germany (1991).

6 R. LANGRETH, "Smart Shovel," Popular Science, Vol. 240, No. 6, pp. 83-85, (1992).

7 D. A. BRADLEY, D. W. SEWARD, and R. H. BRACEWELL, "Control and Operational Strategies for Automatic Excavation," 6th International Symposium on Automation and Robotics in Construction, Vol. 1, pp. 237-244, San Francisco, California (1989).

8 P. K. ALLEN, Robotic Object Recognition Using Vision and Touch, Kluwer Academic Publishers, Boston (1987). 